Document downloaded from:

http://hdl.handle.net/10251/84783

This paper must be cited as:

Legaz-Aparicio, A.; Verdú-Monedero, R.; Larrey-Ruiz, J.; Morales-Sánchez, J.; López-Mir, F.; Naranjo Ornedo, V.; Bernabéu, Á. (2017). Efficient Variational Approach to Multimodal Registration of Anatomical and Functional Intra-Patient Tumorous Brain Data. International Journal of Neural Systems. 27(6):1750014-1-1750014-11. doi:10.1142/S0129065717500149.

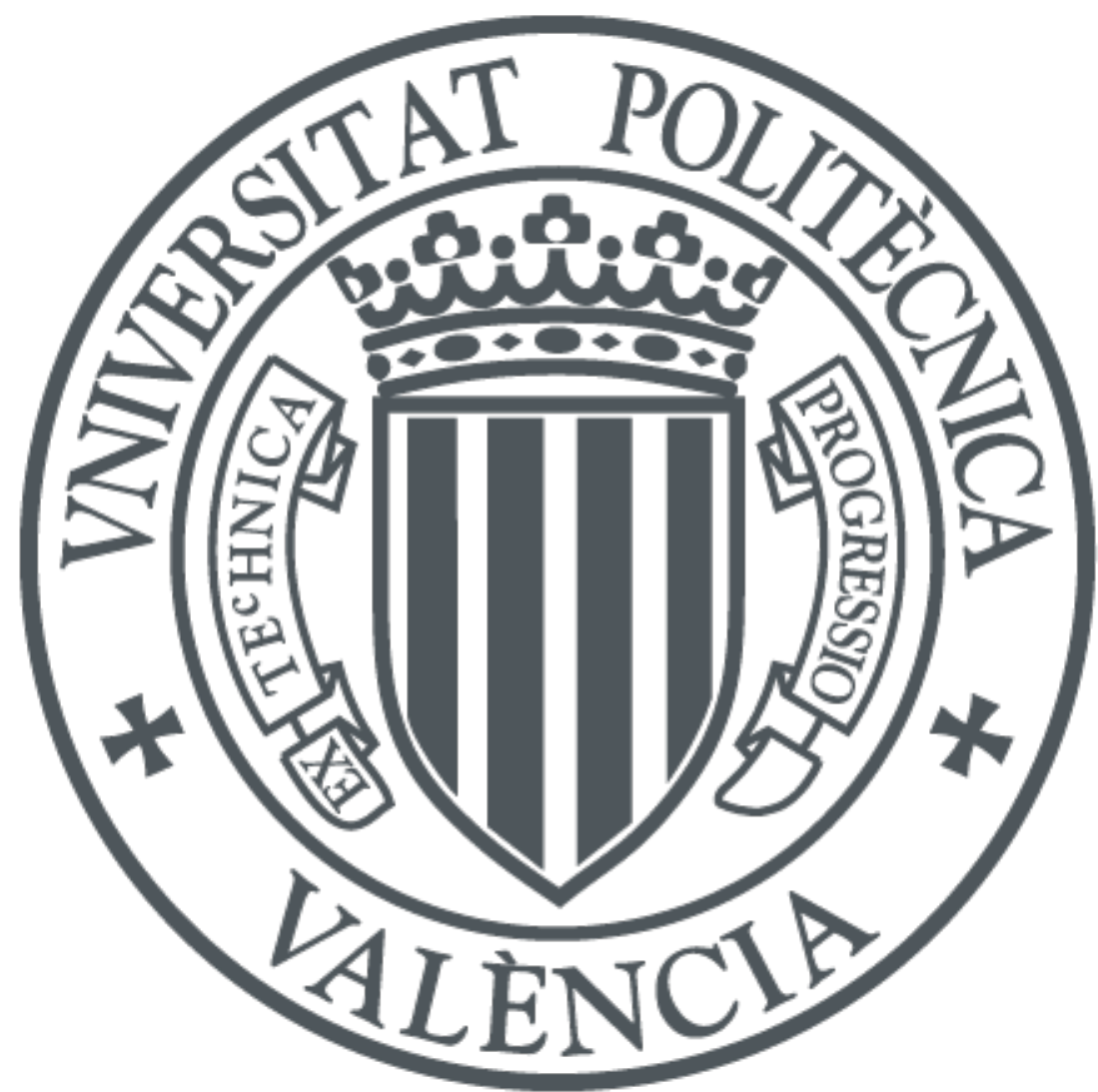

The final publication is available at

http://dx.doi.org/10.1142/S0129065717500149

Copyright World Scientific Publishing

Additional Information 


\title{
EFFICIENT VARIATIONAL APPROACH TO MULTIMODAL REGISTRATION OF ANATOMICAL AND FUNCTIONAL INTRA-PATIENT TUMOROUS BRAIN DATA
}

\author{
ALVAR-GINÉS LEGAZ-APARICIO ${ }^{1}$, RAFAEL VERDÚ-MONEDERO ${ }^{1}$, JORGE LARREY-RUIZ ${ }^{1}$, \\ JUAN MORALES-SÁNCHEZ ${ }^{1}$, FERNANDO LÓPEZ-MIR ${ }^{2}$, VALERY NARANJO ${ }^{2}$, AND ÁNGELA BERNABÉU ${ }^{3}$ \\ ${ }^{1}$ Universidad Politécnica de Cartagena, Plaza del Hospital, 1. Cartagena, 30202, Spain \\ ${ }^{2}$ Universidad Politécnica de Valencia, Camino de Vera s/n, Valencia, 46022, Spain \\ ${ }^{3}$ Inscanner S.L, Unidad de Resonancia Magnética, Avenida de Dénia, 78. Alicante, 03016, Spain
}

\begin{abstract}
This paper addresses the functional localization of intra-patient images of the brain. Functional images of the brain (fMRI and PET) provide information about brain function and metabolism whereas anatomical images (MRI and CT) supply the localization of structures with high spatial resolution. The goal is to find the geometric correspondence between functional and anatomical images in order to complement and fuse the information provided by each imaging modality. The proposed approach is based on a variational formulation of the image registration problem in the frequency domain. It has been implemented as a $\mathrm{C} / \mathrm{C}++$ library which is invoked from a GUI. This interface is routinely used in the clinical setting by physicians for research purposes (Inscanner, Alicante, Spain), and may be used as well for diagnosis and surgical planning. The registration of anatomic and functional intra-patient images of the brain makes it possible to obtain a geometric correspondence which allows for the localization of the functional processes that occur in the brain. Through 18 clinical experiments, it has been demonstrated how the proposed approach outperforms popular state-of-the-art registration methods in terms of efficiency, information theory-based measures (such as mutual information) and actual registration error (distance in space of corresponding landmarks).
\end{abstract}

Keywords: image registration, variational registration, multimodal registration, functional localization, brain imaging, brain registration.

\section{Introduction}

Image registration is the process of finding the optimum geometrical transformation which relates corresponding points of two dataset (images or volumes). ${ }^{1}$ Applied to medical imaging, image registration tries to find the correspondence between datasets obtained at different times or with different acquisition modalities or devices. ${ }^{2,3}$ The registration of functional and anatomic brain images belonging to the same patient is an essential part of the functional localization process, among other applications. ${ }^{4,5}$ Functional images of the brain, such as functional magnetic resonance images (fMRI) or positron emission tomographies (PET), provide information about brain function and metabolism but they do not give detailed structural information and do not provide a specific anatomical location of the functional information. PET is a molecular imaging technique widely used in the clinical setting and in basic research in neurology, cardiology and particularly in oncology due to its outstanding sensitivity and limitless depth of penetration. ${ }^{6,7}$ On the other hand, $\mathrm{fMRI}^{8,9}$ is a noninvasive technique based on the blood oxygenation leveldependent effect (BOLD), which serves as an indirect measure of the neuronal activity. This provides information about brain function as well as the location of functional brain areas (also known as Brodmann areas). ${ }^{10,11}$ Functional images are able to detect brain activated areas but they do not provide anatomical information, hence it is necessary to analyze them along with anatomical images. The common procedure used to more precisely locate specific areas under study within the brain is the registration of the fMRI and PET results (activated brain areas) with high-resolution anatomical images acquired through conventional magnetic resonance images (MRI) and computed tomography (CT). However, CT data presents some limitations such as its low contrast and lack of specificity in the identification and characterization of tumor lesions and some central nervous system structures. ${ }^{12}$ As a consequence, the registration of functional images and MRI is more appropriate ${ }^{13}$. On the other hand, CT scans 
are primarily used for radiotherapy (RT) planning, ${ }^{14}$ where MRI and CT are registered to circumvent the limitations of CT. This type of registration, where datasets show different contrast and intensity levels, is known as multimodal registration. The main goal of the multimodal registration process is to find an unambiguous correspondence between functional and anatomical images of a patient, even if these correspondences are not clearly visible in both imaging modalities. Spatial resolution, signal-to-noise ratio and contrast are lower in functional images than in anatomical images. PET and fMRI images provide very vague and imprecise brain structures. On the other hand, MRI and CT images usually have a high resolution and definition of brain structures.

When dealing with datasets from different modalities, intrinsic deformations caused by different acquisition devices may appear. These deformations are typically non-rigid and present many challenges in the registration task, such as anatomical variability, intensity inhomogeneity (due to bias field) or pathology-induced missing correspondences in pathology-bearing images. However, studies of the brain at different times can show significant differences due to growing tumors or surgical resection. A brain with a (growing) tumor suffers a deformation which usually requires a non-rigid registration approach.

In the last decade, researchers have developed and implemented algorithms and libraries for image registration. Examples of general purpose toolboxes for image registration can be found in Refs. 15-17. Biomechanical and statistical models have also been developed to set up the anatomical atlas as a previous step to its registration with tumor masses, ${ }^{18}$ since the area of the brain affected by a tumor presents a large deformation of the anatomical structure and texture.

In this paper, we present an efficient approach to the registration of anatomical images (MRI and CT) and functional images (fMRI and PET) in order to obtain the correspondence between images of both modalities. The authors deal with specific characteristics of the general framework previously presented in Ref. 19; and more precisely, with its application to an actual clinical scenario through a new $\mathrm{C} / \mathrm{C}++$ implementation. The results are compared with ANTS ${ }^{*}$ and Elastix ${ }^{\dagger, 16}$, two of the most outstanding state-of-the-art registration methods which are publicly available.

\section{Methodology}

Throughout this paper, the datasets to be registered (i.e., reference dataset, $R$, and template dataset, $T$ ) are volumes obtained from different medical studies (CT, MRI, fMRI and PET), $R, T: \mathbb{R}^{3} \rightarrow \mathbb{R}$. Mathematically, the outcome of the registration is a non-rigid displacement field $\mathbf{u}: \mathbb{R}^{3} \rightarrow$ $\mathbb{R}^{3}$ as such, the transformed template is similar to the reference dataset in the geometrical sense, $T(\mathbf{x}-\mathbf{u}(\mathbf{x})) \approx R(\mathbf{x})$, where $\mathbf{u}(\mathbf{x})=\left(u_{1}(\mathbf{x}), u_{2}(\mathbf{x}), u_{3}(\mathbf{x})\right)$ and $\mathbf{x}$ is the spatial position, $\mathbf{x}=\left(x_{1}, x_{2}, x_{3}\right) \in \mathbb{R}^{3}$. This registration problem can be approached in terms of variational calculus, by defining the joint energy functional to be minimized:

$$
J[\mathbf{u}]=D[R, T ; \mathbf{u}]+\alpha S[\mathbf{u}] .
$$

The energy term $D$ measures the distance between the deformed template and the reference dataset; $S$ is a penalty term which acts as a regularizer and determines the smoothness of the displacement field; and $\alpha>0$ weights the influence of the regularization.

The distance measure $D$ is chosen depending on the particular datasets to be registered. When dealing with volumes from different sources or modalities (i.e., in a multimodal registration scenario), information theorybased measures are the most appropriate choice. In this work the opposite of the correlation ratio ${ }^{20}$ (CR) is proposed:

$$
D[R, T ; \mathbf{u}]=-\mathrm{CR}\left[R, T_{\mathbf{u}}\right]=-1+\frac{\mathrm{E}\left\{\operatorname{Var}\left\{T_{\mathbf{u}} \mid R\right\}\right\}}{\operatorname{Var}\left\{T_{\mathbf{u}}\right\}},
$$

where $T_{\mathbf{u}}=T(\mathbf{x}-\mathbf{u}(\mathbf{x}))$ is the deformed template, $\mathrm{E}$ is the mathematical expectation and Var is the variance; the intensities of the datasets are considered as random variables.

The regularization term $S$ gives the smoothness characteristics to the displacement field. Among the most popular regularizers that can be found in the literature ${ }^{21}$ we chose the diffusion term:

$$
S[\mathbf{u}]=\frac{1}{2} \sum_{l=1}^{3} \int_{\psi}\left\|\nabla u_{l}(\mathbf{x})\right\|^{2} d \mathbf{x}
$$

\footnotetext{
* stnava.github.io/ANTs/
} 
where $\psi \subset \mathbb{R}^{3}$ is the domain in which the datasets are supported, and $\nabla$ denotes the gradient operator with respect to the spatial variable $\mathbf{x}$. This regularizing term is designed to penalize oscillating deformations (since it is based on the energy of first-order spatial derivatives), thus being suitable for the registration scenario considered in this work, where only slight misalignments are expected.

As described in Ref. 19, the joint energy functional (1) can be translated into the frequency domain by means of Parseval's theorem. According to this theorem, the value of $J[\mathbf{u}]$ is independent of the domain where it is computed, therefore;

$$
J[\mathbf{u}]=\tilde{J}[\tilde{\mathbf{u}}]=\tilde{D}[R, T ; \tilde{\mathbf{u}}]+\alpha \tilde{S}[\tilde{\mathbf{u}}],
$$

with $\tilde{\mathbf{u}}(\boldsymbol{\omega})=\left(\tilde{u}_{1}(\boldsymbol{\omega}), \tilde{u}_{2}(\boldsymbol{\omega}), \tilde{u}_{3}(\boldsymbol{\omega})\right)$ being the frequency counterpart of the displacement field, $\boldsymbol{\omega}=\left(\omega_{1}, \omega_{2}, \omega_{3}\right)$ being the three-dimensional (3D) variable in the frequency domain, and where both the distance measure $\tilde{D}$ and the regularization term $\tilde{S}$ are now defined in the frequency domain.

From a variational point of view, a necessary condition for a minimizer $\tilde{\mathbf{u}}$ of the joint energy functional (4) is that the first variation of $\tilde{J}[\tilde{\mathbf{u}}]$ in any direction (also known as the Gâteaux derivative) vanishes for all suitable perturbations. This leads to the translation of an Euler-Lagrange (E-L) equation into the frequency domain:

$$
\tilde{\mathbf{f}}(\boldsymbol{\omega})+\alpha \tilde{\mathbf{A}}(\boldsymbol{\omega}) \tilde{\mathbf{u}}(\boldsymbol{\omega})=\mathbf{0},
$$

where $\tilde{\mathbf{f}}(\boldsymbol{\omega})$ is the 3D Fourier transform of the so-called external forces field, $\mathbf{f}(\mathbf{x})$ (please refer to Appendix A, Eqs. (A.1)-(A.2) for a definition of this field), and $\tilde{\mathbf{A}}$ is a diagonal $3 \times 3$ matrix whose elements are scalar functions which implement the spatial derivatives in the frequency domain, ${ }^{22}$ allowing for their computation by means of products:

$$
\tilde{\mathrm{A}}_{k k}(\boldsymbol{\omega})=2 \sum_{l=1}^{3}\left(1-\cos \omega_{l}\right), \quad k=1,2,3 .
$$

Equation (5) provides a stable implementation for the computation of a numerical solution for the displacement field, and in a more efficient way than existing approaches if the 3D fast Fourier transform (FFT) is used..$^{22}$ In order to solve (5) in its current state (i.e., formulated in the frequency domain), a timemarching scheme can be employed, yielding the following equation:

$$
\partial_{t} \tilde{\mathbf{u}}(\boldsymbol{\omega}, t)+\tilde{\mathbf{f}}(\boldsymbol{\omega}, t)+\alpha \tilde{\mathbf{A}}(\boldsymbol{\omega}) \tilde{\mathbf{u}}(\boldsymbol{\omega}, t)=\mathbf{0},
$$

where $\partial_{t} \tilde{\mathbf{u}}(\boldsymbol{\omega}, t)=\left(\partial_{t} \tilde{u}_{1}(\boldsymbol{\omega}, t), \partial_{t} \tilde{u}_{2}(\boldsymbol{\omega}, t), \partial_{t} \tilde{u}_{3}(\boldsymbol{\omega}, t)\right)$. In the steady-state $\partial_{t} \tilde{\mathbf{u}}(\boldsymbol{\omega}, t)=\mathbf{0}$, hence (7) holds (5). Equation (7) can be solved by discretizing time, $t=\xi \tau$, with $\tau>0$ being the time-step and $\xi \in \mathbb{N}$ being the iteration index, and then replacing the time derivative of $\tilde{\mathbf{u}}(\boldsymbol{\omega}, t)$ with its first backward difference. Using the notation $\tilde{\mathbf{u}}^{(\xi)}(\boldsymbol{\omega})=\tilde{\mathbf{u}}(\boldsymbol{\omega}, \xi \tau)$, the following semi-implicit iterative scheme comes out:

$$
\tilde{u}_{l}^{(\xi)}(\boldsymbol{\omega})=\tilde{\mathrm{H}}(\boldsymbol{\omega})\left(\tilde{u}_{l}^{(\xi-1)}(\boldsymbol{\omega})-\tau \tilde{f}_{l}^{(\xi-1)}(\boldsymbol{\omega})\right),
$$

with $l=\{1,2,3\}$, and where $\tilde{\mathrm{H}}(\boldsymbol{\omega})$ is the following lowpass filter:

$$
\tilde{\mathrm{H}}(\boldsymbol{\omega})=\frac{1}{1+\tau \alpha \tilde{\mathrm{A}}_{k k}(\boldsymbol{\omega})} .
$$

It should be noted that all products and divisions in equations (8) and (9) are pointwise. Iteration (8) can be understood as a gradient descent method, which is globally convergent since the joint energy functional (4) to be minimized is a convex quadratic function of the displacement field. According to quadratic modelling, the existence and uniqueness of the solution is therefore guaranteed. Finally, once the algorithm converges, the displacement field in the spatial domain, $\mathbf{u}(\mathbf{x})$, can be obtained as the inverse Fourier transform of its frequency counterpart.

\section{Results}

In this section, the performance of the proposed registration framework is evaluated through 18 experiments. The experiments involve the registration of anatomical (MRI and CT) and functional (fMRI and PET) images of the brain. The aim of these experiments is to show the ability of the registration framework to improve the functional localization of primary tumors in the central nervous system and help to plan the most appropriate treatment. The datasets used in the registration process are based on studies of 10 patients. The MRI and fMRI studies were acquired in a 3T Philips Achieva scanner with a SENSE Neurovascular coil provided by the manufacturer (Philips, The Netherlands); FDG-PET datasets were acquired in a GE Discovery STE 16 scanner (General Electric Medical Systems, Milwaukee, Wisconsin, USA); and CT studies were acquired in a Siemens Sensation 10 and a Siemens 
Table 1. Summary of the studies carried out on each patient.

\begin{tabular}{|c|c|c|c|c|}
\hline Patient Id & Study & Size (voxels) & Resolution & Acquisition device \\
\hline$\# 1$ & MRI & $352 \times 352 \times 200$ & $240 \mathrm{~mm} \times 240 \mathrm{~mm} \times 1 \mathrm{~mm}$ & Philips Achieva 3T \\
\hline$\# 1$ & PET & $128 \times 128 \times 46$ & $271 \mathrm{~mm} \times 271 \mathrm{~mm} \times 3 \mathrm{~mm}$ & General STE 16 \\
\hline$\# 1$ & CT & $512 \times 512 \times 52$ & $228 \mathrm{~mm} \times 228 \mathrm{~mm} \times 3 \mathrm{~mm}$ & Siemens Sensation 10 \\
\hline$\# 2$ & MRI & $432 \times 432 \times 200$ & $250 \mathrm{~mm} \times 250 \mathrm{~mm} \times 1 \mathrm{~mm}$ & Philips Achieva 3T \\
\hline$\# 2$ & fMRI & $64 \times 64 \times 28$ & $230 \mathrm{~mm} \times 230 \mathrm{~mm} \times 4 \mathrm{~mm}$ & Philips Achieva 3T \\
\hline$\# 2$ & PET & $128 \times 128 \times 46$ & $271 \mathrm{~mm} \times 271 \mathrm{~mm} \times 3 \mathrm{~mm}$ & General STE 16 \\
\hline$\# 2$ & $\mathrm{CT}$ & $512 \times 512 \times 52$ & $228 \mathrm{~mm} \times 228 \mathrm{~mm} \times 3 \mathrm{~mm}$ & Siemens Sensation 10 \\
\hline$\# 3$ & MRI & $336 \times 336 \times 200$ & $227 \mathrm{~mm} \times 227 \mathrm{~mm} \times 1.3 \mathrm{~mm}$ & Philips Achieva 3T \\
\hline \#3 & fMRI & $64 \times 64 \times 30$ & $230 \mathrm{~mm} \times 230 \mathrm{~mm} \times 4 \mathrm{~mm}$ & Philips Achieva 3T \\
\hline$\# 3$ & PET & $128 \times 128 \times 30$ & $271 \mathrm{~mm} \times 271 \mathrm{~mm} \times 3 \mathrm{~mm}$ & General STE 16 \\
\hline$\# 4$ & MRI & $512 \times 512 \times 262$ & $255 \mathrm{~mm} \times 255 \mathrm{~mm} \times 1.4 \mathrm{~mm}$ & Philips Achieva 3T \\
\hline$\# 4$ & fMRI & $64 \times 64 \times 27$ & $230 \mathrm{~mm} \times 230 \mathrm{~mm} \times 4 \mathrm{~mm}$ & Philips Achieva 3T \\
\hline$\# 5$ & MRI & $560 \times 560 \times 36$ & $250 \mathrm{~mm} \times 250 \mathrm{~mm} \times 4 \mathrm{~mm}$ & Philips Achieva 3T \\
\hline$\# 5$ & CT & $512 \times 512 \times 52$ & $228 \mathrm{~mm} \times 228 \mathrm{~mm} \times 3 \mathrm{~mm}$ & Siemens Sensation 10 \\
\hline$\# 5$ & PET & $128 \times 128 \times 27$ & $271 \mathrm{~mm} \times 271 \mathrm{~mm} \times 3 \mathrm{~mm}$ & General STE 16 \\
\hline$\# 6$ & MRI & $352 \times 352 \times 200$ & $240 \mathrm{~mm} \times 240 \mathrm{~mm} \times 1 \mathrm{~mm}$ & Philips Achieva 3T \\
\hline$\# 6$ & fMRI & $64 \times 64 \times 26$ & $230 \mathrm{~mm} \times 230 \mathrm{~mm} \times 4 \mathrm{~mm}$ & Philips Achieva 3T \\
\hline$\# 6$ & CT & $512 \times 512 \times 32$ & $265 \mathrm{~mm} \times 265 \mathrm{~mm} \times 5 \mathrm{~mm}$ & Siemens Emotion 16 \\
\hline$\# 6$ & PET & $128 \times 128 \times 46$ & $271 \mathrm{~mm} \times 271 \mathrm{~mm} \times 3 \mathrm{~mm}$ & General STE 16 \\
\hline$\# 7$ & MRI & $256 \times 256 \times 24$ & $320 \mathrm{~mm} \times 320 \mathrm{~mm} \times 4 \mathrm{~mm}$ & Philips Achieva 3T \\
\hline$\# 7$ & PET & $128 \times 128 \times 15$ & $331 \mathrm{~mm} \times 331 \mathrm{~mm} \times 8 \mathrm{~mm}$ & General STE 16 \\
\hline$\# 7$ & fMRI & $64 \times 64 \times 24$ & $230 \mathrm{~mm} \times 230 \mathrm{~mm} \times 4 \mathrm{~mm}$ & Philips Achieva 3T \\
\hline$\# 8$ & MRI & $256 \times 256 \times 24$ & $320 \mathrm{~mm} \times 320 \mathrm{~mm} \times 4 \mathrm{~mm}$ & Philips Achieva 3T \\
\hline$\# 8$ & PET & $128 \times 128 \times 15$ & $331 \mathrm{~mm} \times 331 \mathrm{~mm} \times 8 \mathrm{~mm}$ & General STE 16 \\
\hline$\# 9$ & MRI & $256 \times 256 \times 52$ & $209 \mathrm{~mm} \times 209 \mathrm{~mm} \times 3 \mathrm{~mm}$ & Philips Achieva 3T \\
\hline$\# 9$ & $\mathrm{CT}$ & $512 \times 512 \times 40$ & $215 \mathrm{~mm} \times 215 \mathrm{~mm} \times 3 \mathrm{~mm}$ & Siemens Sensation 10 \\
\hline$\# 10$ & MRI & $256 \times 256 \times 36$ & $209 \mathrm{~mm} \times 209 \mathrm{~mm} \times 4 \mathrm{~mm}$ & Philips Achieva 3T \\
\hline$\# 10$ & $\mathrm{CT}$ & $512 \times 512 \times 40$ & $228 \mathrm{~mm} \times 228 \mathrm{~mm} \times 3 \mathrm{~mm}$ & Siemens Sensation 10 \\
\hline$\# 10$ & fMRI & $64 \times 64 \times 27$ & $230 \mathrm{~mm} \times 230 \mathrm{~mm} \times 4 \mathrm{~mm}$ & Philips Achieva 3T \\
\hline
\end{tabular}

Emotion 16 (Siemens Healthcare GmbH, Germany). These studies were performed as part of the clinical protocol. Table 1 summarizes the studies carried out whereas Table 2 gathers the registration input for all the experiments, which can be classified in three scenarios: registration of MRI and PET, experiments \#1-\#6; registration of fMRI and MRI, experiments \#7-\#12; and registration of MRI and CT, experiments \#13-\#18.

Due to the fact that the datasets in the proposed framework have to share the same spatial range and resolution, the datasets are re-sampled by a non-integer factor through decimation and interpolation steps, thus achieving datasets with the same dimensions, $512 \times 512 \times 128$ voxels.

Fig.1(a), Fig.2(a) and Fig.3(a) display the quality measurements for each experiment. The quality of registration is measured in terms of mutual information (MI). In addition, the numerical results provided by the proposed registration framework are compared with two of the most recent and widely used state-of-art registration methods. In particular, we have chosen Elastix and ANTS for such a comparison. Elastix was the fastest method which provided the best results among the open source methods of image registration which entered in the second phase of Empire10 Challenge. ${ }^{23}$ Moreover, 
Table 2. Summary of the different experiments carried out in the Results section.

\begin{tabular}{crrc}
\hline Experiment & Patient Id & Reference & Template \\
\hline$\# 1$ & 1 & MRI & PET \\
$\# 2$ & 2 & MRI & PET \\
$\# 3$ & 3 & MRI & PET \\
$\# 4$ & 6 & MRI & PET \\
$\# 5$ & 7 & MRI & PET \\
$\# 6$ & 8 & MRI & PET \\
$\# 7$ & 2 & fMRI & MRI \\
$\# 8$ & 3 & fMRI & MRI \\
$\# 9$ & 4 & fMRI & MRI \\
$\# 10$ & 6 & fMRI & MRI \\
$\# 11$ & 7 & fMRI & MRI \\
$\# 12$ & 10 & fMRI & MRI \\
$\# 13$ & 1 & MRI & CT \\
$\# 14$ & 2 & MRI & CT \\
$\# 15$ & 5 & MRI & CT \\
$\# 16$ & 6 & MRI & CT \\
$\# 17$ & 9 & MRI & CT \\
$\# 18$ & 10 & MRI & CT \\
\hline
\end{tabular}

it is a publicly available software package built upon the Insight Toolkit (ITK), ${ }^{15}$ the well-known open source library for medical image processing. Contrary to our method, Elastix is based on a parametric approach, meaning that the number of possible transformations is limited by introducing a parameterization of the transformation to be applied. Rather than implementing a single registration method, Elastix is a collection of parametric intensity-based registration algorithms. For all experiments considered in this work, a non-rigid Bspline transformation model, an adaptive stochastic gradient descent optimizer (ASGD) and the mutual information as similarity measurement yield the best outcome for Elastix. On the other hand, the registration method based on the open-source Advanced Normalizations Tools (ANTS) ranked $2^{\text {nd }}$ in the second phase of the Empire10 challenge and provided the best performance in Ref. 24; however, it took a relatively long time to process a scan pair. In order to appreciate the improvement by the non-rigid registration algorithms versus rigid registration, ANTS has been configured to provide the best affine registration regardless of the computational cost.
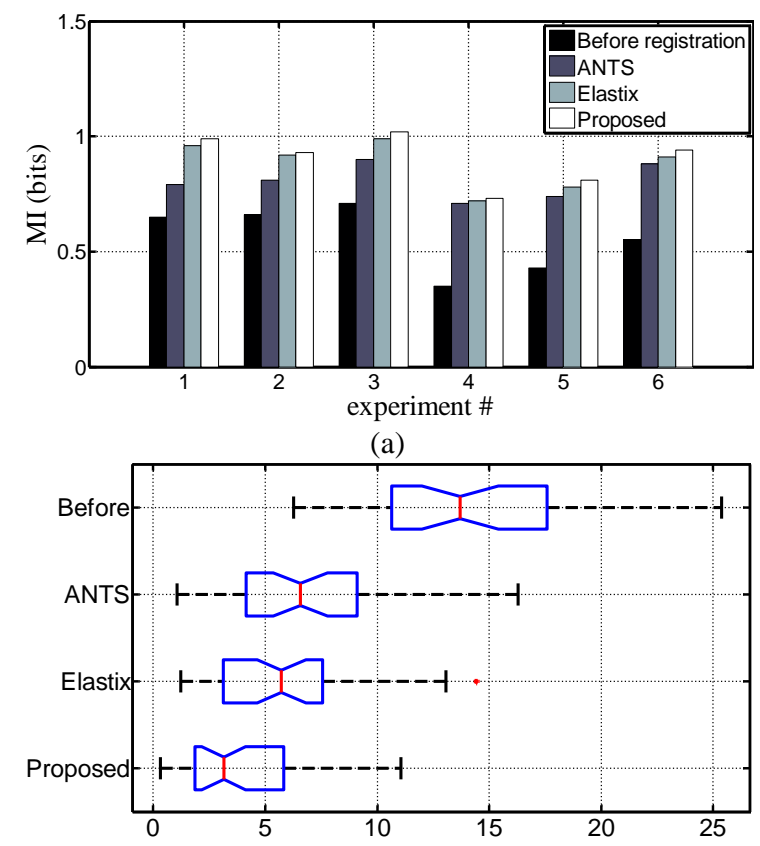

(b)

Fig.1. Registration of MRI and PET. (a) Mutual information in bits. (b) Registration error in $\mathrm{mm}$.

The proposed registration framework shows excellent results in the 18 experiments, which have been distributed equally among three different registration scenarios: MRI-PET, fMRI-MRI, and MRI-CT (see Table 2 for details). For MRI-PET registration (please refer to Fig.1(a)), the proposed method obtains an average improvement of 0.345 bits in terms of mutual information (with \pm 0.05 bits for a $95 \%$ confidence interval of the true mean). For fMRI-MRI and MRI-CT registration scenarios, the achieved mean improvement was 0.292 and 0.429 bits, respectively ( \pm 0.139 bits for a 95\% confidence interval in both cases).

In Fig.1(a), we can see how the proposed method quantitatively outperforms the registration results achieved by Elastix and ANTS, with two exceptions from the MRI-CT registration scenario. Specifically, it is bettered by ANTS in experiments \#2 and by Elastix in experiment \#5. It can be argued that the improvement achieved through the proposed approach is very slight in this scenario. However, there are two issues that should be stressed at this point. First, the datasets to be registered do share a great quantity of information, since most of the structures shown in their respective imaging modalities are common. As a consequence, the resulting registration 


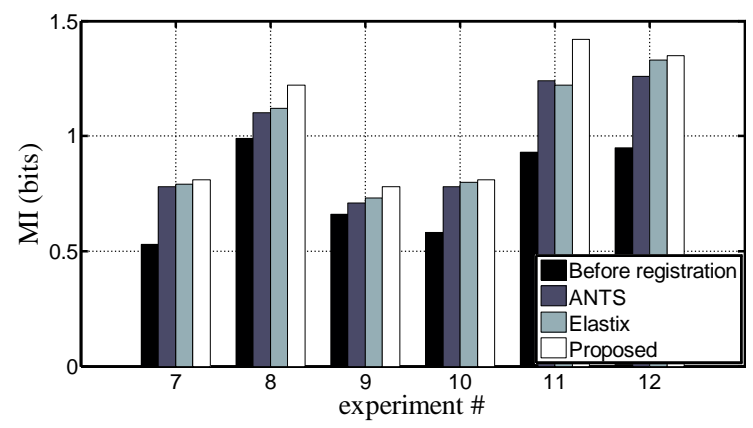

(a)

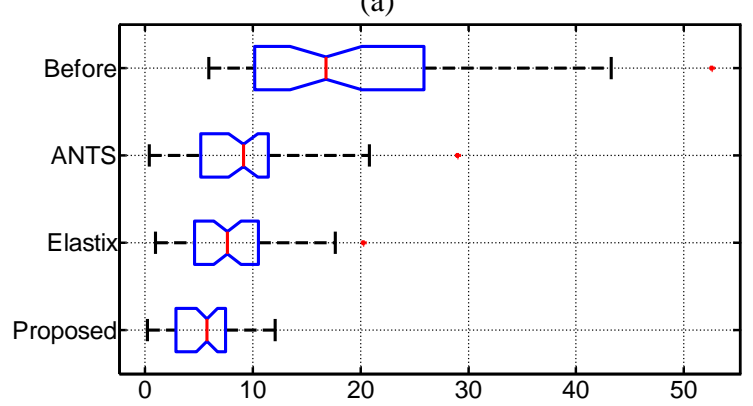

(b)

Fig.2. Registration of MRI and fMRI. (a) Mutual information in bits. (b) Registration error in $\mathrm{mm}$.

scenario is much simpler than in the previous two cases, hence almost every registration algorithm can achieve an acceptable outcome. Second, from the perspective of functional localization of brain images, this scenario is of minor importance, since the datasets to be registered come from purely anatomical imaging modalities. Therefore, the results obtained in the MRI-CT scenario should be considered less relevant than the results reported in Fig.1 and Fig.2, where it is clearly shown how the proposed method outperforms its competitors.

In addition, for a more comprehensive validation, a ground truth was established by an expert in the form of identifiable anatomical locations (landmarks) for all experiments. The actual registration errors were obtained by computing the spatial distance between the corresponding landmarks in the reference and registered template datasets.

Fig.1(b), Fig.2(b) and Fig.3(b) show through box plots the registration errors (in millimeters) achieved by the compared algorithms for the three registration scenarios under consideration: MRI-PET, MRI-fMRI and MRI-CT, respectively. These box plots collect the final spatial distances between corresponding landmarks, along with the median distance error and its statistical significance (notch showing the $95 \%$ confidence interval of the true median).

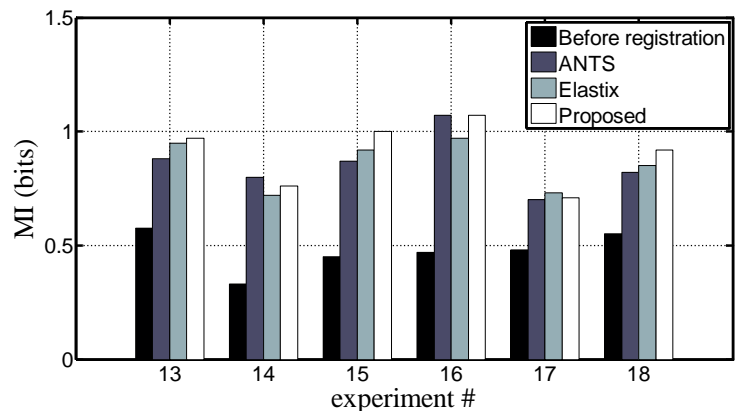

(a)

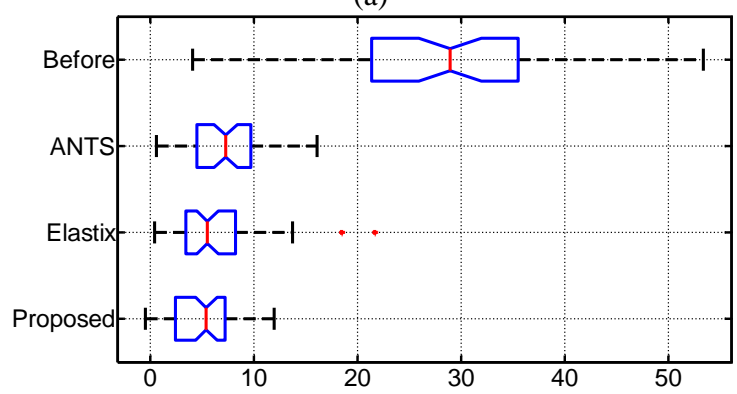

(b)

Fig.3. Registration of MRI and CT. (a) Mutual information in bits. (b) Registration error in $\mathrm{mm}$.

Fig.1(b) has been generated by gathering 42 measurements from 7 selected landmarks for each of the 6 MRI-PET experiments, 5 of these landmarks are placed in order to evaluate the alignment along the brain boundaries while the 2 remaining landmarks are used to measure the alignment of the pathological regions. As can be seen, the proposed method significantly improves on the registration errors of its competitors, reducing the initial median error from $13.69 \mathrm{~mm}$ to a residual median distance between landmarks of $3.15 \mathrm{~mm}$, and the same time attaining the minimum error dispersion.

For both MRI-fMRI and MRI-CT cases (see Fig.2(b) and Fig.3(b)), up to 10 selected landmarks are placed for each of the 6 experiments of the same category (4 landmarks along the brain boundaries, 4 landmarks along the lateral ventricles (butterfly-shaped region) and 2 landmarks along the tumor region). Regarding MRIfMRI registration, comparable conclusions to the previous MRI-PET scenario can be extracted, according to Fig.2(b). Now the median error between corresponding landmarks is reduced from $16.79 \mathrm{~mm}$ to $5.76 \mathrm{~mm}$. Finally, concerning the MRI-CT registration experiments (see Fig.3(b)), the original median distance between corresponding landmarks is shortened from $28.94 \mathrm{~mm}$ to $5.42 \mathrm{~mm}$ (proposed), $5.54 \mathrm{~mm}$ (Elastix), and $7.32 \mathrm{~mm}$ (ANTS). As expected, this time the performance of all evaluated algorithms is much more 
Table 3. Parameter settings of Elastix and ANTS.

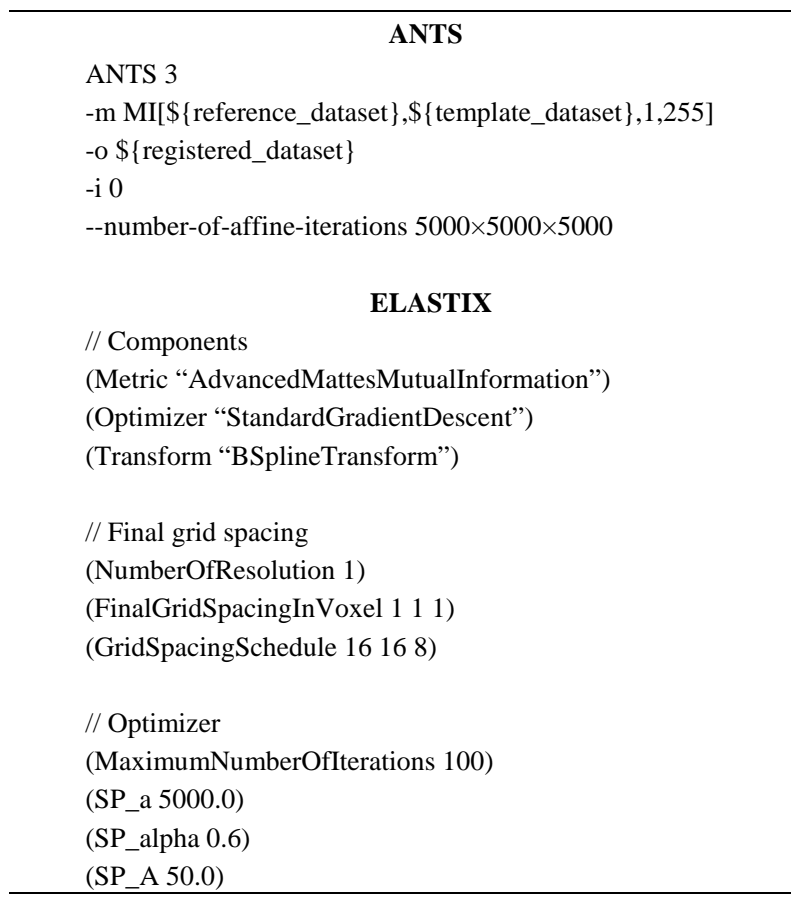

similar, due to the above mentioned issues, although with a slight advantage for the proposed approach in terms of dispersion and outliers.

Regarding the registration parameters, we recommend a common set which achieves a good balance between generalization and performance in all experiments. This way the registration process can be automated and thus the registration framework can be integrated into a fusion application of medical imaging intended to be used by medical personnel. In this paper, the registration parameters of the proposed approach are obtained following the guidelines first introduced in Ref. 25. In particular, the values used for all experiments are $\alpha=30$ and $\tau=1$. From the variational point of view, these values guarantee the best trade-off between similarity of the datasets and smoothness of the solution. As for the number of iterations, a value of $\xi_{\max }=100$ grants convergence in all cases; the cost function stabilizes after 55-60 iterations with our algorithm, and after 50-55 iterations with Elastix. It should be noted that the parameters of Elastix are set in order to ensure fairness in comparison with the proposed method: the same number of maximum iterations, a multimodal similarity measure and only one scale. The parameters of ANTS, on the other hand, are set in order to achieve the
Table 4. Mean timings in seconds for 100 iterations of the proposed registration framework and Elastix for different dimensions of datasets.

\begin{tabular}{ccc}
\hline Size & Proposed & Elastix \\
\hline $128 \times 128 \times 64$ & $60 \mathrm{~s}$ & $140 \mathrm{~s}$ \\
$256 \times 256 \times 128$ & $503 \mathrm{~s}$ & $1120 \mathrm{~s}$ \\
$512 \times 512 \times 128$ & $2084 \mathrm{~s}$ & $4403 \mathrm{~s}$ \\
\hline
\end{tabular}

best affine registration regardless of the computational cost. This affine registration uses a 3 level image pyramid with 5000 iterations at most for each level. The parameter settings of Elastix and ANTS are detailed in Table 3.

In addition to previous measurements, the visual outcomes of some experiments are shown in Fig.4. The first row of Fig.4 illustrates experiment \#4, which deals with the registration of MRI -3D T1-wi gradient-echo sequence- as reference dataset, and 18FDG-PET as template dataset, in a 55 year-old female patient diagnosed with anaplastic oligodendroglioma (patient \#2). The first row of Fig.4 shows how after registration the putative tumor area is located within the right frontal lobe affecting the cingulate gyrus and the genu of the corpus callosum. The pathological area also corresponds to the postsurgical area suggesting tumor recurrance. The outcome of experiment \#7 is displayed in the second row of Fig.4. In this case the reference dataset is the fMRI raw data, whereas the template dataset is 3D T1-wi gradientecho (MRI). The aim of this study is to locate the functional areas related to language processing as well as to determine hemisphere language dominance. Thus, the second row of Fig. 4 shows the registration of the fMRI raw data and the anatomical image; this output is valuable in order to locate the exact position of Broca's area (BA44) in the anatomical image after registration. Results suggest that BA44 is not near the tumor and therefore radiotherapy is proposed. Finally, the third row of Fig.4 shows the registration of MRI (3D T1-wi gradient-echo+Gd) and CT scan of a 31 year-old patient diagnosed with oligodendroglioma II (experiment \#16). The aim of the study is to more precisely locate the tumor region within the right parietal lobe as well as the healthy surrounding structures for better RT planning. The registration outcome shows how the registration of MRI and CT helps in the correct localization of the tumor region.

Regarding the computational time, Table 4 shows a comparison between the proposed registration 


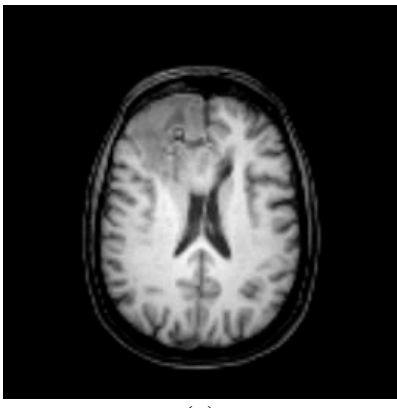

(a)

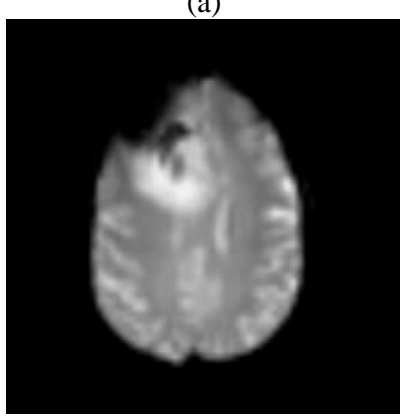

(d)

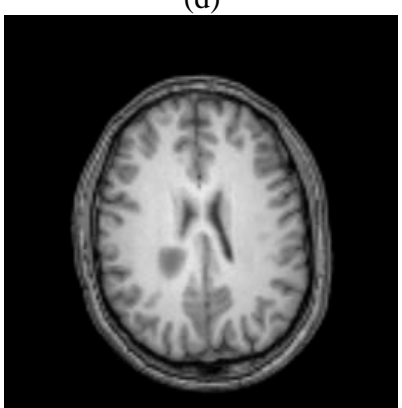

(g)

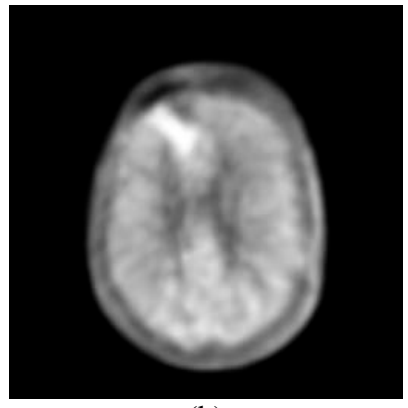

(b)

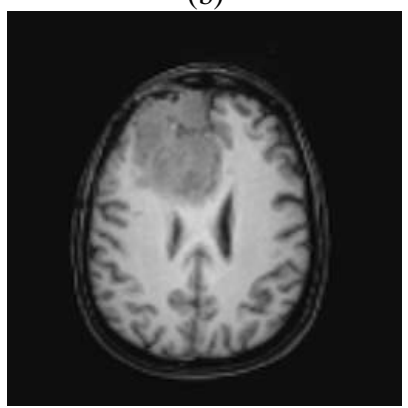

(e)

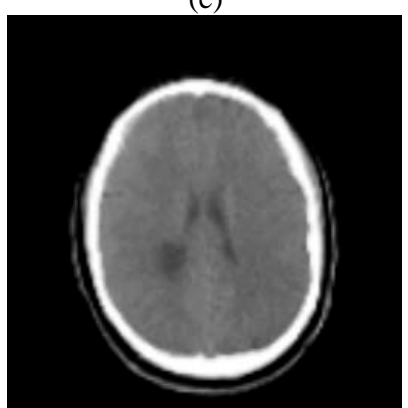

(h)

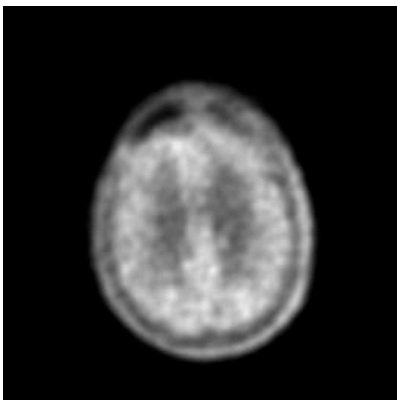

(c)

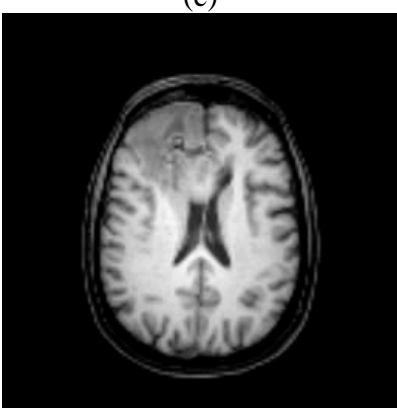

(f)

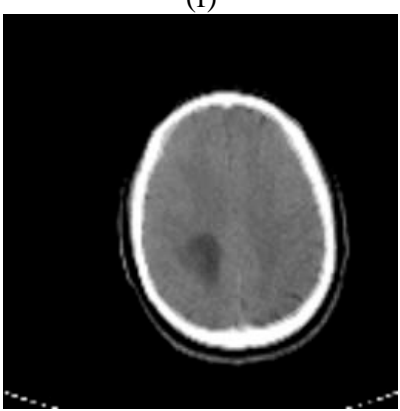

(i)

Fig. 4. First row: Registration of MRI and PET (slice \#75). Second row: Registration of fMRI and MRI (slice \#80). Third row: Registration of MRI and CT (slice \#85). First column: reference dataset. Second column: registered template. Third column: template dataset.

framework and Elastix, both implemented in $\mathrm{C} / \mathrm{C}++$. From the mean timings of the experiments gathered in Table 4, we can conclude that the method introduced in this paper outperforms Elastix in terms of efficiency, since the mean time per iteration of our approach is significantly lower. The overall complexity of each iteration of the resulting algorithm is $\mathrm{O}(\mathrm{N})$ - where $\mathrm{N}$ is the number of voxels in the datasets-, since doubling $\mathrm{N}$ means doubling the computational time. These times were obtained on a PC with Intel Core i5-2500K, 3.3 GHz, 16 GB RAM, running Windows 8.1 (64 bits). Finally, it should be noted that including ANTS in this comparison would not be fair, since ANTS requires a much higher number of iterations in order to obtain the best possible affine registration, which is used in this section to illustrate why a non-rigid approach is needed in the considered clinical scenario.

\section{Discussion and conclusions}

This paper deals with the functional localization of brain images. The method is based on deformable multimodal registration of anatomic (MRI and CT) and functional (fMRI and PET) volumetric brain data. Considering the original misalignment of the brain datasets, the registration algorithm uses the diffusion term as the regularizer. On the other hand, the opposite of the correlation ratio is chosen as the distance term due to the differences in intensity between the imaging modalities to be registered. The suitability of the proposed methodology has been validated by means of 18 illustrative experiments involving different scenarios which consider intra-patient data. When compared with two of the most popular state-of-the-art methods, it has 
been shown that our approach achieves higher values for both the similarity measure considered (mutual information) and the actual registration error (distance in space between corresponding landmarks). Moreover, the results provided by our method are subjectively considered more satisfying after being visually inspected by an expert. However, the main advantage of the proposed registration method is its efficiency. Since it can be implemented in the frequency domain, high computational savings can be achieved if an efficient implementation of the FFT is used. As was proved in previous works which addressed the formulation, efficiency is not incompatible with the quality of the registration results because efficiency is accomplished through the specific implementation of the registration method. This efficiency enables its use in a clinical environment where computational times should be kept as low as possible. Boundary conditions should be mentioned as a possible disadvantage. Due to the use of the three-dimensional discrete Fourier transform, periodic boundary conditions arise naturally when computing a numerical solution for the displacement field. Regardless, when dealing with medical images where the information is typically contained within a uniform background, this difference is hardly noticeable.

It should be noted that comparing the performance of different image registration algorithms is not a trivial task, since each approach has its own set of user-defined parameters, which may heavily influence the final outcome. For instance, if they are tested on datasets with different characteristics (e.g., modality or anatomical region), new optimal parameters need to be determined. In this sense, open challenges are an interesting way of categorizing image registration software packages in a common context. Nevertheless, numerical and visual results, together with their clinical interpretation show the ability and high level of accuracy of the proposed method in obtaining the correspondence between anatomical and functional images of the brain.

In ongoing research, we would like to address the application of our methodology to other medical imaging scenarios, such as the registration of liver CT data under different contrast agent injection. Alternative definitions of the joint functional, including additional energy terms -e.g., a distance measure which incorporates information about external markers or known matching points - are currently being explored within the presented theoretical framework.

\section{Appendix A. External forces field for the CR}

In order to obtain the external forces field of the EulerLagrange equation in the spatial domain, $\mathbf{f}(\mathbf{x})$, the computation of the Gâteaux derivative of the CR-based distance measure $D$ defined in Eq. (2) is required. According to the proof in Ref. 26, the resulting expression for this field is the following:

$$
\mathbf{f}(\mathbf{x})=\frac{1}{N_{\psi}}\left(G_{\beta}(\mathbf{x}) * L_{\mathbf{u}}(\mathbf{x})\right) \nabla T_{\mathbf{u}}(\mathbf{x}),
$$

where $N_{\psi}$ is the number of voxels of the datasets, $G_{\beta}$ is a Gaussian kernel with spread $\beta$, and * denotes the convolution operator. Finally, the function $L_{\mathbf{u}}$ is defined as follows:

$$
L_{\mathbf{u}}=\frac{-2}{\operatorname{Var}\left\{T_{\mathbf{u}}\right\}}\left(\mathrm{E}\left\{T_{\mathbf{u}}\right\}-\mathrm{E}\left\{T_{\mathbf{u}} \mid R\right\}+\mathrm{CR}\left[R, T_{\mathbf{u}}\right]\left(T_{\mathbf{u}}-\mathrm{E}\left\{T_{\mathbf{u}}\right\}\right)\right) .
$$

\section{References}

1. B. Zitová and J. Flusser, Image registration methods: a survey, Image and Vision Computing, 21(11) (2003) 9771000.

2. A. Sotiras, C. Davatzikos and N. Paragios, Deformable medical image registration: a survey, IEEE Trans. on Medical Imaging, 32(7) (2013) 1153-1190.

3. A. Sotiras, Y. Ou, N. Paragios and C. Davatzikos, Graphbased deformable image registration, Handbook of Biomedical Imaging; Methodologies and Clinical Research. Springer. (2015).

4. A. Gholipour, N. Kehtarnavaz, R. Briggs, M. Devous, and K. Gopinath, Brain functional localization: a survey of image registration techniques, IEEE Trans. on Image Processing, 26(4) (2007) 427-451.

5. L. Olson and M.S. Perry, Localization of epileptic foci using multimodality neuroimaging, International Journal of Neural Systems, 23(1) (2013).

6. F-M Lu and Z. Yuan, PET/SPECT molecular imaging in clinical neuroscience: recent advances in the investigation of CNS diseases. Quant Imaging Med Surg. 5(3) (2015) 433-447.

7. S. Surti. Update on time-of-flight PET imaging. $J$ Nucl Med. 56(1) (2015) 98-105.

8. P. Piaggi, D. Menicucci, C. Gentili, G. Handjaras, A. Gemignani, and A. Landi, Adaptive filtering and random variables coefficients for analyzing functional magnetic resonance imaging data, International Journal of Neural Systems, 23(3) (2013).

9. P. Piaggi, D. Menicucci, C. Gentili, G. Handjaras, A. Gemignani, and A. Landi, Singular spectrum analysis and adaptive filtering enhance the functional connectivity analysis of resting state FMRI data, International Journal of Neural Systems, 24(3) (2014). 
10. W. Mier and D. Mier, Advantages in functional imaging of the brain, Front Hum Neurosci. 9(249) (2015).

11. N.K. Logothetis, J. Pauls, M. Augath, T. Trinath and A. Oeltermann, Neurophysiological investigation of the basis of the fMRI signal. Nature 412 (2001) 150-157.

12. G. Sharp, K.D. Fritscher, V. Pekar, M. Peroni, N. Shusharina, H. Veeraraghavan and J. Yang, Vision 20/20: Perspectives on automated image segmentation for radiotherapy. Med Phys. 41(5) (2014).

13. N. Gonçalves, J. Nikkilä, and R. Vigário, Self-supervised MRI tissue segmentation by discriminative clustering, International Journal of Neural Systems, 24(1) (2014).

14. D. Vordermark, Ten years of progress in radiation oncology. BMC Cancer 11(503) (2011).

15. L. Ibáñez, W. Schroeder, L. Ng and J. Cates, The ITK Software Guide, 2nd ed. (Kitware, Clifton Park, NY, 2005).

16. S. Klein, M. Staring, K. Murphy, M.A. Viergever and J.P. Pluim, Elastix: A toolbox for intensity-based medical image registration, IEEE Trans. on Medical Imaging, 29(1) (2010) 196-205.

17. B. Fischer, J. Modersitzki, A unified approach to fast image registration and a new curvature based registration technique. Linear Algebra \& its Applications 308 (2004).

18. A. Mang, A. Toma, T.A. Schuetz, S. Becker and T.M. Buzug, A generic framework for modeling brain deformation as a constrained parametric optimization problem to aid non-diffeomorphic image registration in brain tumor imaging, Methods Inf Med., 51(5) (2012) 429440.

19. J. Larrey-Ruiz, R. Verdú-Monedero, and J. MoralesSánchez, A Fourier domain framework for variational image registration, J. Math. Imaging Vis. 32(1) (2008) 5772.

20. A. Roche, G. Malandian, X. Pennec and N. Ayache, The correlation ratio as a new similarity measure for multimodal image registration, in Proc. MICCAI'98 (LNCS vol. 1496, 1998), pp. 1115-1124.
21. J. Modersitzki, Numerical Methods for Image Registration (Oxford University Press, NY, 2004).

22. R. Verdú-Monedero, J. Larrey-Ruiz, and J. MoralesSánchez, Frequency implementation of the EulerLagrange equations for variational image registration, Signal Processing Letters 15 (2008) 321-324.

23. K. Murphy, B. van Ginneken, J. M. Reinhardt, S. Kabus, K. Ding, X. Deng, K. Cao, K. Du, G. E. Christensen, V. Garcia, T. Vercauteren, N. Ayache, O. Commowick, G. Malandain, B. Glocker, N. Paragios, N. Navab, V. Gorbunova, J. Sporring, M. de Bruijne, X. Han, M. P. Heinrich, J. A. Schnabel, M. Jenkinson, C. Lorenz, M. Modat, J. R. McClelland, S. Ourselin, S. E. A. Muenzing, M. A. Viergever, D. De Nigris, D. L. Collins, T. Arbel, M. Peroni, R. Li, G. C. Sharp, A. Schmidt-Richberg, J. Ehrhardt, R. Werner, D. Smeets, D. Loeckx, G. Song, N. Tustison, B. Avants, J. C. Gee, M. Staring, S. Klein, B. C. Stoel, M. Urschler, M. Werlberger, J. Vandemeulebroucke, S. Rit, D. Sarrut, J. P. W. Pluim, Evaluation of registration methods on thoracic CT: The empire10 challenge, IEEE Trans on Medical Imaging 30(11) (2011) 1901-1920.

24. Y. Ou, H. Akbari, M. Bilello, X. Da, and C. Davatzikos, Comparative evaluation of registration algorithms in different brain databases with varying difficulty: results and insights, IEEE Trans. on Medical Imaging, 33(10) (2014) 2039-2065.

25. J. Larrey-Ruiz and J. Morales-Sánchez, Optimal parameters selection for non-parametric image registration methods, Lecture Notes in Computer Science, 4179 (2006) 564-575.

26. G. Hermosillo, C. Chefd'Hotel and O. Faugeras, A variational approach to multi-modal image matching, Technical Report 4117 (INRIA, 2001). 